\title{
Topic Selection and Structure in the National Atlas of Switzerland
}

\author{
Raphael Vomsattel*, René Sieber, Lorenz Hurni \\ Institute of Cartography and Geoinformation, ETH Zurich, \{vraphael|sieberr|lhurni\}@ethz.ch \\ * Corresponding author
}

Keywords: Atlas Concepts, Topic Selection, Thematic Navigation, National Atlas

\begin{abstract}
:
The establishment of a National Atlas requires a broad view on the thematic content. Traditionally, maps in atlases are accessed by selecting topics from a thematically structured menu. Many important and complex decisions have to be made by map authors regarding the selection and structure of topics which are considered as the essence of an atlas. In the following lines will be described how and why the selected topics have been realized in the Atlas of Switzerland (AoS) and on which concepts they are based on.
\end{abstract}

\section{Thematic Concepts of National Atlases}

A first guideline how to build up a national atlas has been published in 1960 by the international commission on national atlases lead by Salichtchev (Academie des Sciences de 1'URSS). Among a brief historical review, general considerations and mathematical basis the report gives specific recommendations on the content of a national atlas. In the following years most of the national atlas committees orientated on Salichtchev's principles (Ormeling, 1979). Nevertheless, since these days the subjects and objectives have been adjusted to contemporary needs and opportunities. The concepts of national atlases no longer followed a country-specific, but a socio-geographical approach in order to fulfil these complex challenges. The focus is primarily on the human-centred, which requires a problem-oriented and experience-oriented approach.

Today, three different genres of map themes can be distinguished in a national atlas: The first type includes the classical and traditional topics, which were primarily determined under Salichtchev's commission. This includes maps that can be found in almost every national atlas (e.g. geology, population density, etc.). The second type of map deals with current issues that affect people and their environment (e.g. migration flows, desertification, etc.). The third genre includes maps that have been created to be visually and technically attractive. These maps are strongly individual and characterized by the fact that they are more about the implementation of the topic than about its content.

\section{Thematic Structure}

A well elaborated thematic structure is of great importance for an atlas. The thematic navigation is besides spatial navigation, visualisation, analysis and general atlas functions one of the five main functionality groups of a digital atlas (Sieber and Huber, 2007). Therefore, it especially plays a significant role with respect to usability. Maps can be selected using the thematically-arranged menu which are organized in multiple levels. This elaborated hierarchical organisation of the subject categories assists the user not only in navigation but also in maintaining an overview of related map topics. In a highly-interactive atlas combinations of multiple maps topics are required and an adequate thematic structure allows to provide specific and reasonable suggestions to the user.

\section{Thematic Content and Structure in the Atlas of Switzerland}

The very first printed AoS was published in 1961. The primary aim of the atlas was to present the diversity of the country's characteristic structures and processes. As a result, the focus was strongly country-specific. Until the late nineties, 596 maps were released. In the course of an evaluation and the advancing digitalization, a new concept for the future orientation of the National Atlas was elaborated. From then on, a broader range of topics with current and problem-related issues was to be dealt with (Projektgruppe Atlas der Schweiz, 1998).

When the first digital version of AoS was released in 2000, the atlas covered 250 topics in the categories of society, economy, state and politics. The second version published four years later consisted of about 1000 topics. Nature and environment were added to the previously integrated subject categories. The following third and predecessor version of the current atlas was released in 2010 and counted about 2000 topics. Transport, energy and communication have been included to the atlas as further categories. The recent on-line version is in process of being expanded and currently holds about 160 themes. Newly integrated categories are History and Future, Landscape and Space as well as Tourism and Leisure time. In addition, the two categories Portrait of Switzerland and Global Switzerland illustrate characteristic traits of Switzerland. The overall thematic structure is already defined, and we will therefore explain in this contribution which topics and how they have been selected. 
Topics are derived from multiple sources. On the one hand, they are directly embedded in the socio-economic context of the current project. On the other hand, the topics are orientated towards existing cartographic products. For the AoS, previous versions were a fundamental source of information. In addition, there are other national atlases and geoportals which provide an excellent reference for the topics. A further thematic influence, which is becoming increasingly important today, results from interaction with users. User Activity Tracking (UAT) and direct feedbacks help to develop an appropriate and attractive structure of thematic content.

However, the topics from the manifold sources are subject to various limitations, which ultimately have a significant influence on the choice of topics. In principle, they are to be subordinated to conceptual provisions. The politics surrounding a national atlas project and its financing determine the framework conditions. Technical limits for implementation are also decisive and must be taken into account prior to the choice of topic. In addition, data availability and visualisation within the atlas impose certain restrictions.

In the presentation we will explain the selection of topics and their hierarchical organisation in the AoS in more detail. Thereby we will focus on the elaborated thematic concept of the latest version of the AoS and point out which intentions are being pursued. For example, more maps are to be produced on current issues affecting people. Furthermore, it is planned that more flagship maps will be published to highlight the visual and technical possibilities of the atlas. On the other hand, we will disclose alternative access options to maps. Traditionally in atlases, access to maps is mainly via the selection of topics in the menu. It is conceivable that access could be enabled via a specific locality. Based on this, map themes and elements that are relevant for this location could be proposed. A storytelling-based form of implementation could be another approach to access a map. 